\title{
ORIGINAL
}

\section{VALIDACIÓN DE LA ESCALA EHEALTH LITERACY (EHEALS) EN POBLACIÓN UNIVERSITARIA ESPAÑOLA}

\author{
Gema Paramio Pérez (1), Bartolomé Jesús Almagro (2), Ángel Hernando Gómez (3) y José Ignacio \\ Aguaded Gómez (4).
}

(1) Grupo de Investigación Ágora (HUM-648). Universidad de Huelva. Huelva. España.

(2) Área Departamental de Educación. Actividad Física y Deporte. CEU Cardenal Spínola (adscrito a la Universidad de Sevilla). Bormujos. Sevilla. España.

(3) Departamento de Psicología Evolutiva y de la Educación, Universidad de Huelva. Huelva. España.

(4) Departamento de Educación, Universidad de Huelva. Huelva. España.

Financiación. Sin financiación, ni pública ni privada.

Los autores declaran que no existen conflictos de interés.

\section{RESUMEN}

Fundamento: Una de las escalas más empleadas para medir de forma rápida y sencilla la competencia en eSalud es la eHealth Literacy Scale (eHEALS), sin embargo, no existe su validación en castellano. Por ello, el objetivo de este estudio fue adaptar y validar al contexto español la escala eHEALS.

Métodos: Se administró la traducción al español de la escala eHEALS, junto a otras escalas para medir algunos indicadores del bienestar psicológico (autoestima, vitalidad subjetiva y satisfacción con la vida). Se utilizó una muestra de 447 estudiantes universitarios de edades entre los 18 y los 45 años. Se realizó un análisis factorial exploratorio, un análisis factorial confirmatorio, análisis de invarianza, de fiabilidad, de estabilidad temporal y de correlaciones bivariadas.

Resultados: El análisis factorial exploratorio mostró una estructura monofactorial que explicó el $52,55 \%$ de la varianza, con elevados pesos factoriales de sus ítems. Se obtuvo una fiabilidad de 0,87 y una correlación test-retest de 0,78 . El análisis factorial confirmatorio mostró unos índices de ajustes adecuados: $\chi 2=66,60 ; \mathrm{p}=0,00 ; \chi 2 / \mathrm{gl}=4,44$; índice de ajuste comparativo $=0,97) ;$ índice de ajuste incremental $=0,97$; índice tucker-lewis $=0,94$ error de aproximación cuadrático medio $=0,08$; residuo cuadrático medio estandarizado $=0,05$. No hubo diferencias por sexo. En cuanto a la validez de criterio, se obtuvieron correlaciones estadísticamente significativas y positivas e entre 0,12 y 0,16 con tres indicadores del bienestar psicológico (autoestima, vitalidad subjetiva y satisfacción con la vida).

Conclusiones: La versión española de la eHEALS testada ha mostrado ser una escala válida y fiable para medir la competencia en eSalud en el alumnado universitario.

Palabras clave: eSalud. Alfabetización en salud. Internet. Cuestionario. Validación. Estudiantes.

Correspondencia

Gema Paramio Pérez.

Grupo de Investigación Ágora (HUM-648)

Facultad de Ciencias de la Educación, Universidad de Huelva

Avenida Tres de Marzo, s/n

21071 Huelva

gema.paramio@dpsi.uhu.es

\section{ABSTRACT}

\section{Validation of the eHealth Literacy Scale (eHEALS) in Spanish University Students}

Background: One of the scales most used to measure quickly and easily eHealth Literacy is the eHealth Literacy Scale (eHEALS); however, there was no validation of this scale in Spanish. Therefore, the aim of this study was to adapt and validate the eHealth Literacy Scale (eHEALS) to the Spanish context.

Methods: Spanish translation of the scale eHEALS was administered along with other scales to measure some indicators of psychological wellbeing (self-esteem, subjective vitality and life satisfaction). A sample of 447 university students aged between 18 and 45 years was selected. An exploratory factor analysis, confirmatory factor analysis, analysis of invariance, reliability, temporal stability and bivariate correlations were performed.

Results: Exploratory factor analysis revealed a monofactorial structure that explained $52.55 \%$ of variance, with high factor loadings of the items. Reliability of 0.87 and test-retest correlation of 0.78 was obtained. The confirmatory factor analysis showed appropriate adjustments indices: $\chi^{2}=66.60 ; \mathrm{p}=0.00 ; \chi^{2} / \mathrm{df}=4.44 ;$ comparative fit inde $\mathrm{x}=0.97$; incremental fit index $=0.97$; tucker lewis index $=0.94$; root mean square of approximattion $=0.08$; standardized root mean square residual $=0.05$. The questionnaire was invariant by gender. Regarding the criterion validity, a statistically significant and positive correlations between 0.12 and 0.16 with three indicators of psychological wellbeing was obtained (self-esteem, subjective vitality and life satisfaction).

Conclusions: The spanish version of the eHEALS tested in this work has shown to be a valid and reliable scale to measure eHealth competence in university students.

Keywords: eHealth. Health literacy. Internet. Questionnaire. Validation. Students. 


\section{INTRODUCCIÓN}

Las tecnologías de la información y la comunicación juegan un papel cada vez más importante en la salud de las personas y están teniendo un profundo impacto en la asistencia sanitaria ${ }^{1}$.

Internet tiene el potencial suficiente para mejorar de forma efectiva la prestación sanitaria capacitando y educando a los consumidores, apoyando en la toma de decisiones, permitiendo la interacción entre consumidores y profesionales, ayudando en la formación e investigación y reduciendo las desigualdades en salud ${ }^{1-3}$.

Si bien es cierto que los modelos de atención tradicionales están dando paso progresivamente a otros más modernos, en los que el papel más activo es asumido por el paciente, también lo es que los profesionales médicos sobrestiman sistemáticamente el nivel de alfabetización en salud de los pacientes proporcionándoles información excesivamente compleja. Esperan de ellos que puedan llevar a cabo tareas más específicas de forma independiente, estando ligadas cada vez más a nuevos dispositivos tecnológicos. En esta línea, la gestión de la salud a través de portales o aplicaciones móviles supone nuevos retos para los usuarios ${ }^{2}$. De hecho, la iniciativa promovida por el Sistema Nacional de Salud en España para facilitar materiales informativos, que impulsen la participación ciudadana en las decisiones y los cuidados de salud es ya una realidad ${ }^{3}$. Un claro ejemplo de ello es el portal PyDEsalud: Participa y decide sobre tu salud $^{3}$ (PyDEsalud.com), web médica de gran impacto socioeconómico creada para mejorar el conocimiento y la participación activa de personas afectadas por enfermedades crónicas $^{3}$.

En respuesta a esta necesidad de acceso y utilización eficaz de la información surge el concepto de alfabetización mediática en salud, que es "la capacidad de buscar, encontrar, comprender y evaluar la información de salud a partir de fuentes electrónicas y aplicar los conocimientos adquiridos para abordar o resolver un problema de salud"4. La alfabetización mediática en salud está compuesta por una serie de competencias generales, como son la alfabetización tradicional (lectura, escritura y aritmética), la alfabetización de los medios de comunicación (habilidades de análisis de los medios de comunicación), la alfabetización de la información (búsqueda de información y la comprensión) y una serie de competencias más específicas, que incluyen la alfabetización informática (habilidades de Tecnología de la Información y Comunicación (TIC), la alfabetización de la salud (comprensión del conocimiento de la salud) y la divulgación de la ciencia (proceso de la ciencia y de resultados) $)^{5}$.

La adaptación de las aplicaciones tecnológicas en salud se hace necesaria para reducir las desigualdades y maximizar los beneficios de los pacientes con todos los niveles de alfabetización. En este sentido, cobra especial importancia la forma de medir las competencias de los usuarios para poder implementar programas de capacitación tanto para estos como para los profesionales de la salud.

A pesar de que la alfabetización en salud ha sido identificada como un objetivo de salud pública para el siglo XXI y un reto importante para la salud mundial ${ }^{6}$, el hecho de medir el nivel de alfabetización en eSalud no es un proceso sencillo. La mayoría de estudios empíricos usan la eHealth Literacy Scale ${ }^{7}$ y lo complementan con algún instrumento para medir la alfabetización en salud ${ }^{5}$ o con una prueba de procedimientos que requiere que los participantes lleven a cabo una serie de operaciones específicas con el ordenador e internet ${ }^{8}$.

La escala de aptitud para utilizar la sanidad electrónica (eHEALS) fue desarrollada por Norman y Skinner en 2006, con el fin de hacer frente a la necesidad de evaluar la alfabetización mediática en salud en una amplia gama de poblaciones y contextos ${ }^{7}$. En concreto, la $e H E A L S$ es una herramienta basada en la percepción subjetiva que tienen los sujetos 
acerca de las habilidades y conocimientos que poseen sobre la eSalud o sanidad electrónica.

La eHEALS está traducida a varios idiomas y validada en diversos contextos culturales. Hasta ahora se han publicado cuatro versiones validadas: en población holandesa ${ }^{9}$, en población china ${ }^{10}$, en población japonesa ${ }^{11}$ y la última, publicada recientemente, con una pequeña muestra de universitarios colombianos $^{12}$. Algunos de estos trabajos han relacionado la competencia en eSalud con la cantidad del uso de internet para darle validez de criterio a la escala ${ }^{9}$, con los conocimientos de informática, incluyendo la capacidad de utilizar un procesador de textos o el uso de la web para buscar información ${ }^{10}$ o con las características de las búsquedas de información sobre salud ${ }^{11}$. En esta línea, existen estudios que han mostrado una relación positiva entre la alfabetización en salud y el bienestar psicológico $^{13}$. Por lo que para darle validez de criterio a la escala eHealth podría relacionarse con indicadores del bienestar psicológico como pueden ser la satisfacción con la vida, la vitalidad subjetiva y la autoestima.

El objetivo de este estudio fue adaptar y validar al contexto español la escala eHealth Literacy Scale.

\section{SUJETOS Y MÉTODO}

Diseño. La presente investigación corresponde a un estudio instrumental ${ }^{14}$, ya que se adaptó y validó al contexto español la eHealth Literacy Scale (eHEALS).

Procedimiento. Se realizó una traducción inversa de los ítems del eHEALS ${ }^{7}$, es decir, se tradujeron los ítems al castellano y, posteriormente, un traductor ajeno al grupo de investigación los volvió a traducir al inglés, observando una gran similitud con el cuestionario original en habla inglesa. A continuación se llevó a cabo el estudio piloto de la escala traducida al español con un grupo de 40 universitarios de edades comprendidas entre los 18 y los 35 años, los cuales manifestaron una buena comprensión de los ítems.
Se contactó con el representante legal de la universidad, decanos, directores de departamento y profesores de las diferentes facultades para pedirles su colaboración. La administración de las escalas tuvo lugar en presencia del investigador principal, para poder explicar de forma breve cómo responder a los cuestionarios. Además, se informó que la participación en el estudio era voluntaria. El tiempo aproximado de cumplimentación fue de 15 minutos. La recogida de datos tuvo lugar entre enero y abril de 2013.

Participantes. La muestra del estudio estuvo compuesta por un total de 447 estudiantes, de los cuales 290 eran mujeres y 156 hombres, de edades comprendidas entre los 18 y los 45 años (media=21,90; desviación típica=3,90). El alumnado universitario que compuso la muestra pertenecía a las facultades de Ciencias de la Educación y Enfermería de la Universidad de Huelva. En concreto, los participantes estudiaban las siguientes titulaciones: Grado en Enfermería (84), en Ciencias de la Actividad Física y del Deporte (86), en Educación Primaria (54), en Educación Infantil $(\mathrm{n}=28)$, en Educación Social (57), en Psicología (40), Licenciatura en Psicología (54) y en Psicopedagogía (44). Se llevó a cabo la selección de las facultades y de las titulaciones atendiendo a un muestreo intencional o por conveniencia.

Instrumentos. Se empleó un cuestionario que estaba compuesto por la escala que se quería validar y por tres escalas que median tres indicadores del bienestar psicológico, para poder relacionar la competencia en eSalud con el bienestar psicológico y darle validez de criterio a la versión en castellano de la eHEALS. A continuación se describen con más detalle las escalas:

Escala de competencia en eSalud. Se empleó la traducción al español del eHealth Literacy Scale (eHEALS) ${ }^{7}$. Esta escala está compuesta por un total de ocho ítems (tabla 1). Las respuestas fueron recogidas en una escala tipo Likert cuyos rangos de puntuación oscilaban desde 1 (muy en desacuerdo) 
hasta 5 (muy de acuerdo). Se estudiaron su consistencia interna, su estabilidad temporal, así como el resto de propiedades psicométricas del instrumento (análisis factorial exploratorio y análisis factorial confirmatorio). La estabilidad temporal se evaluó a través de la realización del test-retest. Para ello la escala eHEALS se administró a 30 estudiantes de $2^{\circ}$ curso del Grado en Psicología. Transcurridos 30 días del llenado del test, se llevo a cabo el retest.

Escala de Satisfacción con la Vida. Se utilizó la versión en castellano ${ }^{15}$ de la Satisfaction with Life Scale (SWLS) ${ }^{16}$, que mide la satisfacción con la vida como un proceso de juicio cognitivo. Las respuestas fueron puntuadas con una escala tipo Likert que oscilaba entre 1 (muy en desacuerdo) y 5 (muy de acuerdo).

Escala de Vitalidad Subjetiva. Se utilizó la versión en castellano ${ }^{17}$ de la Subjective Vitality Scale (SVS) ${ }^{18}$. Sus ítems se refieren a la energía percibida, el entusiasmo y los sentimientos de vivacidad y se utilizó una escala tipo Likert con un rango de 1 (totalmente en desacuerdo) a 5 (totalmente de acuerdo). Se utilizaron los seis ítems directos, tal como se propone en investigaciones anteriores ${ }^{19}$.

Escala de Autoestima de Rosenberg. Se empleó la versión validada al castellano ${ }^{20} \mathrm{de}$ la Rosenberg Self-Esteem Scale ${ }^{21}$ (RSES). La RSES se compone de 10 ítems que evalúan la autoestima. Las respuestas estaban puntuadas en una escala tipo Likert, con un rango de puntuación que va de 1 (totalmente en desacuerdo) a 4 (totalmente de acuerdo). Los puntos 1, 3, 4, 7 y 10 están formulados en positivo, y los ítems 2, 5, 6, 8, y 9 negativamente. La consistencia interna obtenida fue de 0,82 .

Análisis de datos. Se realizaron los siguientes análisis: factorial exploratorio, factorial confirmatorio, análisis de invarianza, de fiabilidad, de estabilidad temporal y de correlaciones bivariadas. Se realizó un análisis factorial exploratorio de componente principales con el objetivo de estudiar la es- tructura factorial de la escala. Previamente al análisis se calculó la medida de adecuación muestral de Kaiser-Meyer-Olkin (KMO) y el test de esfericidad de Bartlett. Para el análisis de la fiabilidad del instrumento se empleó el cálculo del coeficiente alfa de Cronbach. La estabilidad temporal se midió a través de la realización del test-retest. El cuestionario se administró a 30 estudiantes de $2^{\circ}$ curso del Grado en Psicología. Transcurridos 30 días se realizó el retest. Para tratar de confirmar la estructura factorial obtenida se llevó a cabo un análisis factorial confirmatorio. En este se trató de testar el ajuste de los datos al modelo. Para ello se utilizaron los índices de ajuste más comunes: $\chi^{2}, \chi^{2} / \mathrm{gl}$, Comparative Fit Index (CFI), Incremental Fit Index (IFI), Tucker Lewis Index (TLI), Root Mean Square of Approximattion (RMSEA) y Standardized Root Mean Square Residual (SRMR). Para comprobar que la estructura factorial de la versión española del eHEALS era invariante por sexo se realizó un análisis multigrupo. Este tipo de análisis compara el modelo sin restricciones con una serie de modelos con invarianza en diferentes parámetros. Por último, para comprobar la validez de criterio se calcularon las correlaciones bivariadas entre la competencia en eSalud y tres indicadores del bienestar psicológico (satisfacción con la vida, vitalidad subjetiva y autoestima).

\section{RESULTADOS}

Análisis factorial exploratorio. El índice KMO mostró un valor de 0,865 y el test de esfericidad de Bartlett resultó estadísticamente significativo $\left(\chi^{2}=1651,209 ; p<0,001\right)$. Estos valores indican que la realización de un análisis factorial era posible. En el análisis factorial exploratorio de componentes principales se obtuvo un autovalor de 4,20 y una varianza total explicada de 52,55\% (tabla 1). Tras la realización del análisis se comprobó que los 8 ítems se agrupaban en un único factor: competencia o aptitud en eSalud.

Análisis de consistencia interna. El valor del coeficiente alfa de Cronbach fue de 0,87. 


\section{Tabla 1}

\section{Análisis factorial exploratorio del eHEALS}

\begin{tabular}{|c|c|}
\hline Ítems & Factor \\
\hline 1. Conozco qué recursos sobre salud están disponibles en Internet & 0,712 \\
\hline 2. Sé dónde puedo encontrar recursos útiles sobre salud en Internet & 0,803 \\
\hline 3. Sé cómo puedo encontrar recursos útiles sobre salud en Internet & 0,813 \\
\hline 4. Sé cómo utilizar Internet para encontrar respuestas a mis cuestiones sobre salud & 0,807 \\
\hline 5. Sé cómo utilizar la información sobre salud que encuentro en Internet para que me ayude & 0,749 \\
\hline 6. Tengo las habilidades necesarias para evaluar los recursos sobre salud que encuentro en Internet & 0,645 \\
\hline 7. Puedo distinguir los recursos de salud de alta calidad de los recursos de salud de baja calidad que se encuentran en Internet & 0,745 \\
\hline 8. Tengo confianza a la hora de utilizar la información de Internet para tomar decisiones sobre salud & 0,643 \\
\hline Autovalor & 4,20 \\
\hline
\end{tabular}

\begin{tabular}{|c|c|c|c|c|c|c|c|c|c|c|}
\hline & & álisis mul & igrup & de la & $\begin{array}{l}\text { la } 2 \\
\text { Ivar }\end{array}$ & za fac & rial & sexo & & \\
\hline Modelos & $\chi^{2}$ & $\begin{array}{c}\text { Grados de } \\
\text { libertad }\end{array}$ & $\chi^{2} / \mathrm{gl}$ & $\Delta \chi^{2}$ & $\Delta$ gl. & CFI & IFI & TLI & RMSEA & SRMR \\
\hline Modelo 1 & 119,21 & 30 & 3,97 & - & - & 0,95 & 0,95 & 0,90 & 0,08 & 0,06 \\
\hline Modelo 2 & 123,80 & 37 & 3,35 & 4,58 & 7 & 0,95 & 0,95 & 0,92 & 0,07 & 0,07 \\
\hline Modelo 3 & 124,49 & 38 & 3,28 & 5,28 & 8 & 0,95 & 0,95 & 0,92 & 0,07 & 0,07 \\
\hline Modelo 4 & 147,33 & 51 & 2,89 & & 21 & 0,94 & 0,94 & 0,94 & 0,06 & 0,07 \\
\hline
\end{tabular}

Modelo 1: sin restricciones; Modelo 2: pesos de medida invariantes; Modelo 3: covarianzas estructurales invariantes; Modelo 4: residuos de medida invariantes; CFI: Comparative Fit Index; IFI: Incremental Fit Index; TLI: Tucker Lewis Index; RMSEA: Root Mean Square of Approximattion; SRMR: Standardized Root Mean Square Residual

Estabilidad temporal. Se encontró una correlación test-retest de 0,78 .

Análisis factorial confirmatorio. Tras la realización de un primer análisis factorial confirmatorio, se comprobó que los índices de ajustes no fueron apropiados $\left[\chi^{2}=278,96\right.$; $\mathrm{p}=0,00 ; \quad \chi^{2} / \mathrm{gl}=13,95 ; \quad \mathrm{CFI}=0,84 ; \quad \mathrm{IFI}=0,84$; TLI $=0,78 ; \quad$ RMSEA $=0,17 ; \quad$ SRMR $=0,08]$. Los índices de modificación señalaron que al correlacionar los errores entre los ítems 6 у 7,5 у 6, 4 у 5, 4 у 6, 5 у 7 (figura 1) los índices de ajustes mejoraban hasta considerarse adecuados: $\chi^{2}=66,60 ; p=0,00 ; \chi^{2}$ $\mathrm{gl}=4,44 ; \quad \mathrm{CFI}=0,97 ; \quad \mathrm{IFI}=0,97 ; \quad \mathrm{TLI}=0,94$; $\mathrm{RMSEA}=0,08 ; \mathrm{SRMR}=0,05$.

Análisis de invarianza. Los resultados indicaron que los cuatro modelos comparados presentaban unos índices de ajuste adecuados. Además, no se encontraron diferencias significativas entre el modelo sin restriccio- nes y los modelos con invarianza en los pesos de medida $\left(\Delta \chi^{2}=4,58 ; \Delta \mathrm{gl}=7 ; \mathrm{p}=0,71\right)$, en las covarianzas estructurales $\left(\Delta \chi^{2}=5,28 ; \Delta \mathrm{gl}=8\right.$; $\mathrm{p}=0,73)$ y en los residuos de medida $\left(\Delta \chi^{2}=\right.$ $28,12 ; \Delta \mathrm{gl}=21 ; \mathrm{p}=0,14)$. Estos resultados suponen un fuerte apoyo para afirmar la existencia de invarianza por sexo (tabla 2).

En la tabla 3 se presentan los estadísticos descriptivos de cada una de las variables del estudio, así como las correlaciones bivariadas. En el análisis de correlación se obtuvo que la competencia en eSalud correlacionaba de forma positiva y estadísticamente significativa con la satisfacción con la vida, con la vitalidad subjetiva y con la autoestima, aunque con un coeficiente de correlación bajo en los tres casos (entre 0,12 y 0,16 ). Por su lado, los tres indicadores del bienestar psicológico correlacionaron de forma positiva y estadísticamente significativa entre ellos con un coeficiente de Pearson entre 0,47 y 0,53. 


\section{Tabla 3}

Estadísticos descriptivos y correlaciones de las variables

\begin{tabular}{|l|c|c|c|c|c|c|c|c|}
\hline \multicolumn{1}{|c|}{ Variables } & Rango & Media & $\begin{array}{c}\text { Desviación } \\
\text { típica }\end{array}$ & $\begin{array}{c}\boldsymbol{\alpha} \text { de } \\
\text { Cronbach }\end{array}$ & $\mathbf{1}$ & $\mathbf{2}$ & $\mathbf{3}$ & $\mathbf{4}$ \\
\hline 1. Competencia en eSalud & $1-5$ & 3,26 & 0,74 & 0,87 & - & $0,16^{*}$ & $0,16^{*}$ & $0,12^{*}$ \\
\hline 2. Satisfacción con la vida & $1-5$ & 4,08 & 0,67 & 0,81 & - & - & $0,53^{*}$ & $0,47^{*}$ \\
\hline 3. Vitalidad subjetiva & $1-5$ & 3,99 & 0,61 & 0,83 & - & - & - & $0,49^{*}$ \\
\hline 4. Autoestima & $1-4$ & 3,35 & 0,46 & 0,82 & - & - & - & - \\
\hline${ }^{*} \mathrm{p}<0,01$ & &
\end{tabular}

\section{Figura 1}

Análisis factorial confirmatorio de la versión en castellano de la escala $e H E A L S$.

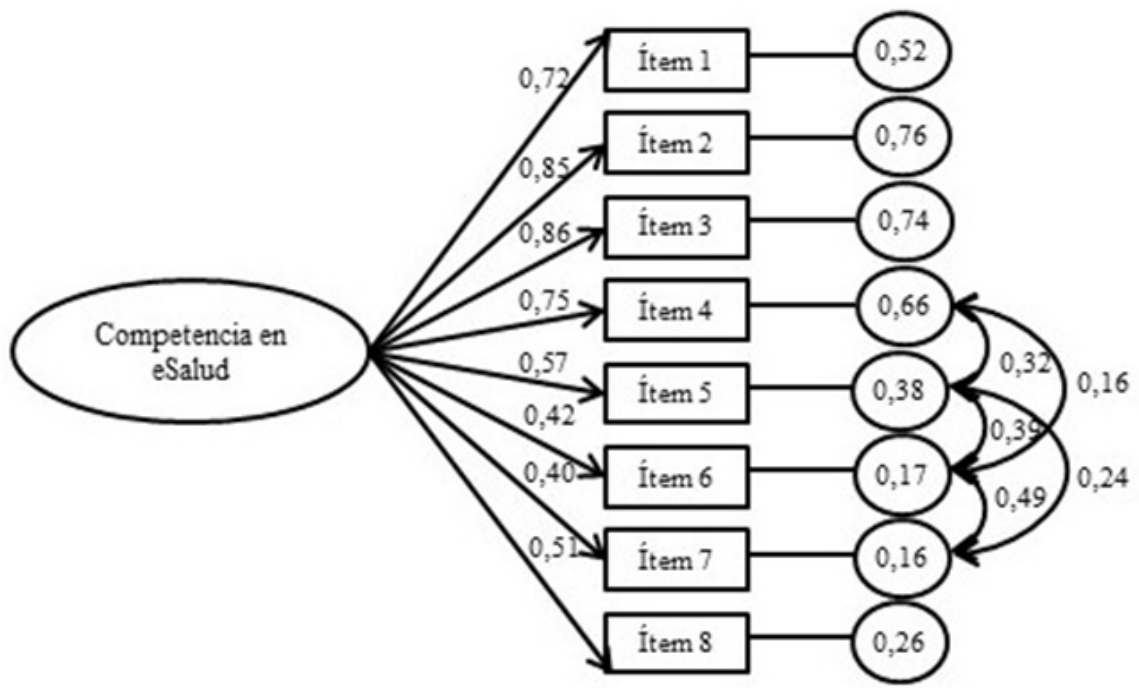

La elipse representa la variable latente y los rectángulos los diferentes ítems. Todos los parámetros están estandarizados y fueron estadísticamente significativos $(\mathrm{p}<0,05)$. Las varianzas error se muestran en pequeños círculos

\section{DISCUSIÓN}

El análisis factorial exploratorio mostró que el único factor de la escala estaba compuesto por 8 ítems, coincidiendo con lo establecido en la escala original (eHEALS) ${ }^{7}$ y en las otras versiones ${ }^{9-11}$ exceptuando la colombiana $^{12}$. Los resultados del análisis de consistencia interna y estabilidad temporal también fueron satisfactorios. Asimismo, se realizó un análisis factorial confirmatorio puesto que en las validaciones previas sólo la versión japonesa $^{11}$ lo había calculado, encontrando índices de ajustes adecuados. No obstante, se tuvie- ron que correlacionar los errores de medida de los ítems 4, 5, 6 y 7 para que los valores de los índices de bondad de ajustes mejorasen. Por su parte, los resultados del análisis multigrupo indican que la estructura factorial de la escala de competencia en eSalud es invariante en función del sexo. Esta última prueba no había sido testada anteriormente y, sin duda, supone una fortaleza más en el proceso de validación de la escala.

Por otro lado, los resultados del análisis de correlación mostraron una relación positiva entre la competencia en eSalud y las tres medi- 
das del bienestar psicológico. En este sentido, algunos estudios relacionan positivamente la alfabetización en salud con el bienestar físico y psicológico ${ }^{13}$, con una mejora del estado de salud autopercibido ${ }^{22}$, con el cumplimiento de las prescripciones médicas, la motivación, la confianza en sí mismo y la resiliencia individual a la adversidad ${ }^{6}$. Sin embargo, parece que falta evidencia empírica para demostrar los beneficios de la eSalud ${ }^{23,24}$.

En cuanto a las correlaciones entre las otras tres variables utilizadas para medir el bienestar psicológico de los universitarios, se obtuvieron asociaciones positivas entre la vitalidad subjetiva, la satisfacción con la vida y la autoestima similares a las obtenidas en otros estudios con estudiantes universita$\operatorname{rios}^{25-27}$. De este modo, se sigue poniendo de manifiesto la influencia de estas tres variables entre ellas, aunque la satisfacción con la vida, al ser un constructo más global, es la variable que parece ser predicha tanto por la autoesti$\mathrm{ma}^{28,29}$ como por la vitalidad subjetiva ${ }^{18}$.

Parece evidente que un buen manejo de la eSalud podría tener consecuencias positivas. De hecho, la búsqueda de información sobre salud en internet está creando un nuevo perfil de paciente con más responsabilidad y participación en los aspectos relacionados con su salud $^{30}$, es decir, con mayor empoderamiento. En este sentido, el uso de internet para tareas de promoción y prevención de la salud, para un mejor conocimiento y gestión de la enfermedad, para compartir experiencias en foros o redes sociales de pacientes, etcétera, puede ser una importante herramienta para complementar el trabajo de los profesionales de la salud $^{12}$.

La evolución de las TIC y el uso masivo de internet en nuestra sociedad desarrollada posibilita el acceso a un mayor volumen de información sobre salud que puede facilitar que los ciudadanos se encuentren desbordados y realicen una demanda de servicios sanitarios irracional e innecesaria, que podría entorpecer el funcionamiento del sistema de salud y la relación entre profesional sanitario y paciente e incluso podría tener un impacto negativo en su propia salud ${ }^{31}$. Esto no hace más que evidenciar la importancia de la formación en eSalud, así como de la medición de este constructo para tratar de conocer la aptitud en eSalud de diferentes poblaciones.

A modo de resumen, el análisis de los datos indicó: 1) una estructura monofactorial, con elevados pesos factoriales de sus ítems; 2) niveles satisfactorios de consistencia interna y de estabilidad temporal tras 30 días de la administración del eHEALS; 3) un ajuste del modelo propuesto adecuado; 4) que la estructura factorial de la versión española del eHEALS era invariante por género; y 5) una adecuada validez de criterio, ya que la competencia en eSalud correlacionó de forma positiva y estadísticamente significativa con la satisfacción con la vida, la vitalidad subjetiva y la autoestima del alumnado.

Por tanto, en base a los resultados obtenidos, se puede concluir que la versión española de la $e H E A L S$ es una escala válida y fiable para medir la competencia en eSalud en el alumnado universitario.

Este trabajo presenta algunas limitaciones que deberán ser subsanadas o tenidas en cuenta en futuros estudios. En primer lugar, para obtener unos índices de ajuste aceptables en el análisis factorial confirmatorio fue necesario correlacionar algunos errores (ítems 4, 5, 6 y 7). Sería interesante comprobar si sigue persistiendo este problema en futuras investigaciones, puesto que podría indicar la necesidad de modificar alguno de los ítems. En segundo lugar, con un cuestionario autoadministrado lo que medimos es la percepción que tiene el sujeto sobre su competencia en eSalud. De hecho, puede que la persona que se está evaluando se sobrestime o se subestime, por lo que sería interesante en futuros estudios testar las respuestas del cuestionario (al menos de un porcentaje de la muestra) con otras pruebas supervisadas por expertos para comprobar la veracidad o exactitud de la estimación. 
En definitiva, se concluye que la versión española de la escala eHEALS es una herramienta sencilla, válida y fiable para medir la competencia o aptitud en eSalud en el contexto educativo universitario. No obstante, son necesarias más investigaciones que traten de corroborar los resultados obtenidos en otras poblaciones y con diferentes análisis estadísticos, puesto que la validación es un proceso que no se puede limitar a un solo estudio.

\section{AGRADECIMIENTOS}

Queremos agradecer a todos los docentes y al alumnado de la Universidad de Huelva que tan amablemente se prestaran a colaborar en el presente estudio.

\section{BIBLIOGRAFÍA}

1. Powell JA, Darvell M and Gray JAM. The doctor, the patient and the world-wide web: how the internet is changing healthcare. J R Soc Med. 2003;96:74-6.

2. Bates DW, Bitton A. The future of health information technology in the patient-centered medical home. Health Aff (Millwood). 2010; 29:614-21. DOI: 10.1377/hlthaff.2010.0007

3. Perestelo-Péreza L, Pérez-Ramos J, Abt-Sacks A, et al. Promoción de la participación ciudadana en cuidados de salud a través de PyDEsalud.com. Gac Sanit. 2013;27:466-7.

4. Norman CD, Skinner HA. eHealth literacy: essential skills for consumer health in a networked world. J Med Internet Res. 2006; 8:e9.

5. Collins SA, Currie LM, Bakken S, et al. Health literacy screening instruments for eHealth applications: a systematic review. J Biomed Inform. 2012;45:598-607.

6. Nutbeam D. Health literacy as a public health goal: a challenge for contemporary health education and communication strategies into the 21 st century. Health Promot Int. 2000;15:259-67.

7. Norman, CD, Skinner HA. eHEALS: The eHealth Literacy Scale. J Med Internet Res. 2006; 8:e27.

8. Xie B. Older adults, e-Health literacy, and collaborative learning: An experimental study. Journal of the American Society for Information Science and Technology. 2001;62:933-46.
9. Van der Vaart R., Van Deursen AJ, Drosaert, CHC, et al. Does the eHealth Literacy Scale (eHEALS) measure what it intends to measure? Validation of a dutch version of the eHEALS in two adult populations. J Med Internet Res. 2011;13:e86.

10. Koo M, Norman CD, Chang H-M. Psychometric evaluation of a chinese version of the eHealth Literacy Scale (eHEALS) in school age children. Int Electron J Health Educ. 2012;15:29-36.

11. Mitsutake S, Shibata A, Ishii K, et al. Developing Japanese version of the e-Health Literacy Scale (eHEALS). Nihon Koshu Eisei Zasshi. 2011;58:36171.

12. Rojas DF, Useche B. (2013). Alfabetización digital en salud: un análisis del constructo en la escala "eHealth Literacy Scale- eHeals" traducida al español. RevistaeSalud.com [revista electrónica]. 2013 [consultado el 16/02/2014]; Disponible en: http://revistaesalud.com/index.php/revistaesalud/article/view/639

13. Tokuda Y, Doba N, Butler JD, et al. Health literacy and physical and psychological wellbeing in Japanese adults. Patient Educ Couns. 2009;75: 411-7.

14. Montero I, León OG. A guide for naming research studies in Psychology. Int $\mathrm{J}$ Clin Health Psychol. 2007;7:847-62.

15. Atienza FL, Pons D, Balaguer I, et al. Propiedades psicométricas de la escala de satisfacción con la vida en adolescentes. Psicothema. 2000;12:314-9.

16. Diener E, Emmons R, Larsen RJ, et al. The Satisfaction With Life Scale. J Pers Assess. 1985; 49: 71-5.

17. Balaguer I, Castillo I, García-Merita M, et al. Implications of structured extracurricular activities on adolescent's well being and risk behaviors: Motivational mechanisms. Granada: 9th European Congress of Psychology; 3-8 Jul 2005.

18. Ryan RM, Frederick CM. On energy, personality and health: Subjective vitality as a dynamic reflection of well-being. JPers. 1997;65:529-65.

19. Bostic TJ, Rubio DM, Hood M. A validation of the subjective vitality scale using structural equation modeling. Soc Indic Res. 2000;52:313-24.

20. Martín-Albo J, Núñez JL, Navarro, JG, et al. The Rosenberg Self-Esteem Scale: Translation and Validation in University Students. Span J Psychol. 2007; 10:458-67.

21. Rosenberg M. Society and the adolescent self-image. (Rev. ed.). Middeltown, CT: Wesleyan University Press. 1989. p.347. 
22. Lee SY, Arozullah AM, Cho, YI. Health literacy, social support, and health: a research agenda. Soc Sci Med. 2004;58:1309-1321.

23. Black AD, Car J, Pagliari C, et al. The impact of eHealth on the quality and safety of health care: a systematic overview. PLOS Med. 2011;8:e1000387.

24. Dedding C,Van Doorn R, Winkler L, et al. How will e-health affect patient participation in the clinic? A review of e-health studies and the current evidence for changes in the relationship between medical professionals and patients. Soc Sci Med. 2011;72:49-53.

25. Castillo I, Molina-García J. Adiposidad corporal y bienestar psicológico: efectos de la actividad física en universitarios de Valencia, España. Rev Panam Salud Publica. 2009;26:334-40.

26. Molina-García J. Un estudio sobre la práctica de actividad física, la adiposidad corporal y el bienestar psicológico en universitarios [tesis doctoral]. Valencia: Universidad de Valencia; 2004.

27. Fernández-Ozcorta EJ. Factores motivacionales y su relación con la práctica de actividad física en el alumnado universitario de Huelva [tesis doctoral]. Huelva: Universidad de Huelva; 2013.

28. Moreno-Murcia JA, Vera JA. Modelo causal de la satisfacción con la vida en adolescentes de educación física. Rev Psicodidáctica. 2011;16:367-80.

29. Rey L, Extremera N, Pena M. Inteligencia emocional percibida, autoestima y satisfacción con la vida en adolescentes. Psicosoc Interv. 2011;20:227-34.

30. Ferguson T, Frydman G. The first generation of epatients. BMJ. 2004;328:1148-9.

31. Lupiáñez-Villanueva F. Salud e internet: más allá de la calidad de la información. Rev Esp Cardio. 2011;64:849-50. 


\begin{tabular}{|c|c|c|c|c|c|}
\hline \multicolumn{6}{|l|}{$\begin{array}{l}\text { Anexo 1 } \\
\text { Versión española de la escala eHealth Literacy (eHEALS) }\end{array}$} \\
\hline 1. Conozco qué recursos sobre salud están disponibles en Internet & 1 & 2 & 3 & 4 & 5 \\
\hline 2. Sé dónde puedo encontrar recursos útiles sobre salud en Internet & 1 & 2 & 3 & 4 & 5 \\
\hline 3. Sé cómo puedo encontrar recursos útiles sobre salud en Internet & 1 & 2 & 3 & 4 & 5 \\
\hline 4. Sé cómo utilizar Internet para encontrar respuestas a mis cuestiones sobre salud & 1 & 2 & 3 & 4 & 5 \\
\hline 5. Sé cómo utilizar la información sobre salud que encuentro en Internet para que me ayude & 1 & 2 & 3 & 4 & 5 \\
\hline 6. Tengo las habilidades necesarias para evaluar los recursos sobre salud que encuentro en Internet & 1 & 2 & 3 & 4 & 5 \\
\hline $\begin{array}{l}\text { 7. Puedo distinguir los recursos de salud de alta calidad de los recursos de salud de baja calidad que se } \\
\text { encuentran en Internet }\end{array}$ & 1 & 2 & 3 & 4 & 5 \\
\hline 8. Tengo confianza a la hora de utilizar la información de Internet para tomar decisiones sobre salud & 1 & 2 & 3 & 4 & 5 \\
\hline 1: Completamente en desacuerdo. 2: Algo en desacuerdo. 3: Indeciso. 4: Algo de acuerdo. 5: Completar & & & & & \\
\hline
\end{tabular}

